\title{
Bio-compatible Piezoresistive Pressure Sensing Skin Sleeve for Millimetre-Scale Flexible Robots: Design, Manufacturing and Pitfalls
}

\author{
Piotr Wasylczyk, Filip Ozimek, Manish K. Tiwari, Lyndon da Cruz* , and Christos Bergeles*
}

\begin{abstract}
Safe interactions between humans and robots require the robotic arms and/or tools to recognize and react to the surrounding environment via pressure sensing. With small-scale surgical interventions in mind, we have developed a flexible skin with tens of pressure sensing elements, designed to cover a $5 \mathrm{~mm}$ diameter tool. The prototype uses only biocompatible materials: soft silicones, carbon powder and metal wires. The material performance, sensing element, manufacturing technology, and the readout electronics are described. Our prototype demonstrates the feasibility of using this technology in various intervention scenarios, from endoscopic navigation to tissue manipulation. We conclude by identifying research directions that maximise the potential of the proposed technology.
\end{abstract}

\section{INTRODUCTION}

During robot-assisted medical interventions, robotic arms and end-effectors/tools usually encounter contact forces from the surrounding tissues. While most surgical procedures rely on imaging in guiding the tools, information on the pressure exerted could provide complementary information and reduce the possibility of inadvertent tissue damage. Figure 1a shows a concept of a $5 \mathrm{~mm}$ diameter flexible robotic arm (concentric tube robot), covered with a flexible pressure sensing skin comprising tens of pressure sensors (tactels). The tactels, connected via an array of electrodes, read out the external forces acting on the robot on its way to the operating area inside the body. We present the manufacturing process of developing such flexible skin with piezoresistive pressure sensors, that may cover a robotic arm or tools such as endoscopes and provide force information (see Fig. 1b).

While continuous pressure sensors have been successfully demonstrated [1], most flexible pressure sensor arrays use a grid of capacitance [2], piezoelectric [3] or piezoresistive [4] elements to measure the spatial and temporal distribution of external forces. Piezoresistive sensors usually have an arrays of tactels embedded on or within a soft carrier membrane. Most of the devices demonstrated to date have planar geometry and tens to hundreds of tactels on a millimetre-spaced grid (for a review, see [5], [6]). The tactels can be made of a soft material (such as carbon-loaded silicone rubber) [7], [8] or micro-channels/chambers filled with ionic liquids [9] or eGaIn a metal alloy that remains liquid at room (and body)

This research was supported by an ERC Starting Grant [714562].* denotes equal contribution.

P. Wasylczyk, M. K. Tiwari, and L. da Cruz are with the Wellcome/EPSRC Centre for Interventional and Surgical Sciences, UCL, UK. F. Ozimek is with Mullard Space Science Laboratory, UCL, UK. P Wasylczyk is additionally with Faculty of Physics, University of Warsaw, Poland. L. da Cruz is additionally with Moorfields Eye Hospital, London, UK. C. Bergeles is with the School of Biomedical Engineering and Imaging Sciences, Kings College London, UK (e-mail: christos.bergeles@kcl.ac.uk). (a)

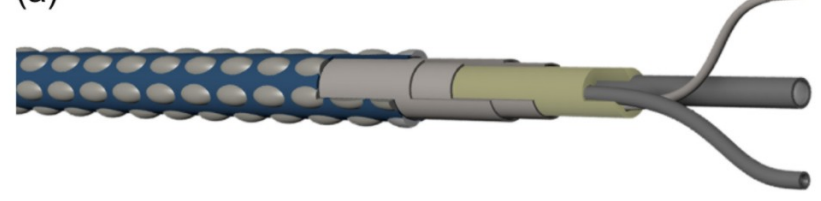

(b)

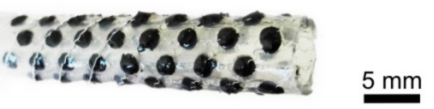

Fig. 1. Tubular pressure-sensitive soft, stretchable skin. (a) Illustration of a concentric tube soft robotic arm covered with a pressure sensor array. (b) Prototype sensor with $8 \times 8$ tactile pixel array and wire electrodes embedded in a soft silicone carrier membrane; the outer diameter of the tube is $7 \mathrm{~mm}$.

temperature [10], [11]. While eGain may offer sophisticated sensing geometries (such as measuring pressure and shear forces simultaneously with the same tactel [12]), it is unlikely to be approved for medical use inside the body.

The membranes supporting the pressure sensing elements are typically made of soft silicone rubber or polyimide films with the electrodes in the form of patterned metal coatings [13], [14], conducting inks [15], embedded metal wires [16], ITO layers [17] or conducting fabric [18], [19]. Making the pressure sensing skin in the form of a tube, rather than a flat membrane, requires a different approach for electrode configuration, as the electrical connections can only be accessed from one end of the tube. For the pressure sensing skin to be useful for flexible instruments, it must be stretchable and this sets several challenges to the carrier membrane, the electrodes and the sensors themselves.

A pressure sensor array for surgical applications should:

1) be scalable in the number of sensing elements most prototypes have up to few tens of tactels,

2) be scalable in the sensing element size and spacing from millimetre scale for typical endoscopy tools, down to tens of microns for e.g. intraocular devices,

3) have a large pressure sensing range ideally extending from a fraction of a pascal $(\mathrm{Pa})$ to a few hundred $\mathrm{Pa}$ to approach the performance of a human skin,

4) have fast response (and recovery) time tens of ms at most to be applicable for real-time interactions [20].

\section{TUBULAR TACTILE SENSOR - FROM PIEZORESISTIVE} SOFT MATERIAL TO FLEXIBLE TUBULAR SLEEVE

\section{A. Evaluation of soft piezoresistive silicone mixtures}

Silicone rubbers are bio-compatible and, in combination with carbon powders, can be the material of choice for 
piezoresistive pressure sensing elements in medical applications [21], [22], [23], [24], [25]. Their piezoresistive behaviour is rather complex - the compressive matrix model assumes the constant carbon volume and the silicone volume decreasing with pressure, resulting in decreasing electrical resistivity as more contacts are created between the conductive particles. On the other hand, the conductive paths may be broken by the silicone matrix deformation, resulting in the opposite effect (i.e. the resistivity increasing with pressure) [18].

For our piezoresistive senor prototype, a two-part silicone (Gumosil AD-1, Silikony Polskie, $30^{\circ} \mathrm{Sh}$ A, $650 \%$ elongation at break) was mixed 1:10 w/w with the platinum catalyst for 1-2 minutes. Five carbon powders at various concentrations were added to the silicone and mixed for 1-2 minutes (see Table 1): graphene nano-platelets, carbon black, acetylene carbon black, and carbon nanofibers. $6 \times 6 \times 5 \mathrm{~mm}$ blocks were cast in a laser-cut poly(methyl methacrylate) (PMMA) mould with glass bottom and cured at room temperature overnight. Each block had a $0.2 \mathrm{~mm}$ diameter copper wire electrode embedded near one surface. The silicone/carbon powder block constitutes the model tactel.

To characterise the tactel's resistivity, the resistance between the wire electrode and a metal plate pressed against the opposite side of the tactel was measured. The results, summarized in Table 1, show the complexity of the material behaviour: the resistance of the samples for the same carbon concentration vary from infinity (i.e. above hundreds of $\mathrm{M}$ ) to hundreds of ohms. In most cases the resistance decreases with increasing pressure, but in one sample it does the opposite.

For the carbon powders we tested, $10 \% \mathrm{w} / \mathrm{w}$ concentration is near the limit of how much powder can be mixed into the silicone at $15 \% \mathrm{w} / \mathrm{w}$ the mixture cannot be cast and the cured rubber loses the elasticity. Carbon black from Alfa Aesar at $10 \% \mathrm{w} / \mathrm{w}$ was chosen for the sensor array prototype: it has the resistance in the range of a few $\mathrm{k}$, well suited for the readout electronics, is sufficiently thin to cast and the resulting material maintains the elasticity of the silicone.

TABLE I

PIEZORESISTIVE SILICONE-CARBON MIXTURES

\begin{tabular}{|c|l|c|c|}
\hline No & w/w \% added carbon & $\mathrm{R}(\Omega)$ & $\Delta \mathrm{R}$ \\
\hline 1 & $\begin{array}{l}10 \% \quad \text { graphene nano- } \\
\text { platelets, 5 } \mu \mathrm{m}, \quad 80 \mathrm{~m}^{2} / \mathrm{g}\end{array}$ & $\infty$ & - \\
& $\begin{array}{l}\text { (Sigma, 900409) } \\
5 \% \text { carbon black, (Alfa Ae- }\end{array}$ & $\infty$ & - \\
3 & $\begin{array}{l}\text { sar, H30253) } \\
10 \% \text { carbon black (Alfa Ae- } \\
\text { sar, H30253) }\end{array}$ & $1-10 \mathrm{k}$ & $\downarrow$ \\
4 & $\begin{array}{l}10 \% \text { graphene nano-platelets } \\
25 \mu \mathrm{m}, 130 \quad \mathrm{~m}^{2} / \mathrm{g} \text { (Sigma, }\end{array}$ & $1-10 \mathrm{M}$ & $\uparrow$ \\
5 & $\begin{array}{l}900413) \\
10 \% \quad \text { carbon nanofibers } \\
6\end{array}$ & $\begin{array}{l}\text { (Sigma, 719781) 100-1000 } \\
10 \% \text { acetylene carbon black } \\
\text { (Alfa Aesar, 45527) }\end{array}$ & $\downarrow$ \\
\hline
\end{tabular}

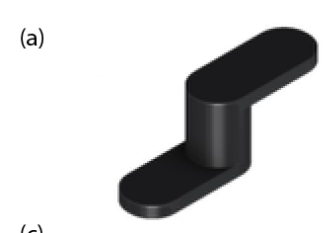

(b)
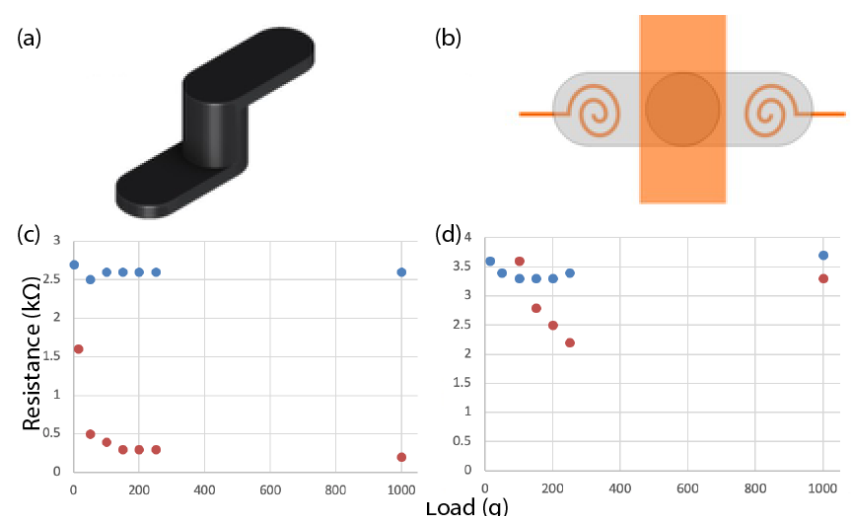

(d) 4

Fig. 2. Resistance vs load measured in different configurations to asses the influence of the electrode contact with two types of silicones. (a) The conductive silicone rubber sample tactel in the form of a cylinder with flaps on both ends. (b) The configuration with wire electrodes in the flaps (outside the compression zone, marked in orange); top view. Measured resistance vs load for two silicones acid cure 151 type silicone glue (c) and platinum catalyst AD-1 (d) with the outside metal plate electrodes pressed against the cylinder ends (red circles) and wire electrodes ouside the compression zone (blue squares).

\section{B. Evaluation of a single tactel - electrode contact effects}

The simplest tactel is a block or cylinder of a piezoresistive material with electrodes at the top and bottom surfaces. Experimenting with conducting silicones we realized that not only the rubber conductivity, but also the resistance of the contacts between the electrodes and the piezoresistive material affects the overall electrical response of the tactel upon pressure. This effect has not been taken into account in previous studies in few cases only has it been noted that the position of the electrodes can affect the tactel response [18]. What we showcase below is that embedding the electrodes within the piezoresistive components actually leads to scewed measurements and should be avoided.

To study these effects we cast cylindrical samples $(3.9 \mathrm{~mm}$ in diameter, $4.3 \mathrm{~mm}$ in height) with two flaps $(8 \mathrm{~mm}$ long, $1 \mathrm{~mm}$ thick) extending to the sides at both ends of the cylinder (Fig. 2a). The wire electrodes were embedded either at the ends of the cylinder (i.e. in the compression zone) or in the flaps. In the first experiment we used two silicone rubbers: the platinum catalyst AD-1 and acid cure 151 adhesive sealant, which reacts with atmospheric moisture to cure, to estimate how the metal electrode contacts contribute to the overall resistivity of the model tactel. The results, plotted in Fig. 2c and 2d indicate that with the wire electrodes outside the compression zone, the tactel resistance changes very little (less than 10\%) with the applied load up to $1000 \mathrm{~g}$ that corresponds to $840 \mathrm{kPa}$ in the silicone cylinder.

Conversely, with the metal plate electrodes pressed against the cylinder, the resistance decreases monotonically up to $250 \mathrm{~g}(210 \mathrm{kPa})$. These results indicate that for both silicones the changes in the rubber-metal contract resistance for the electrodes applied on the tactel surface (not embedded in the conductive rubber) affect the tactel conductivity to much higher degree than the changes of the carbon-loaded silicone resistivity. 
(a)

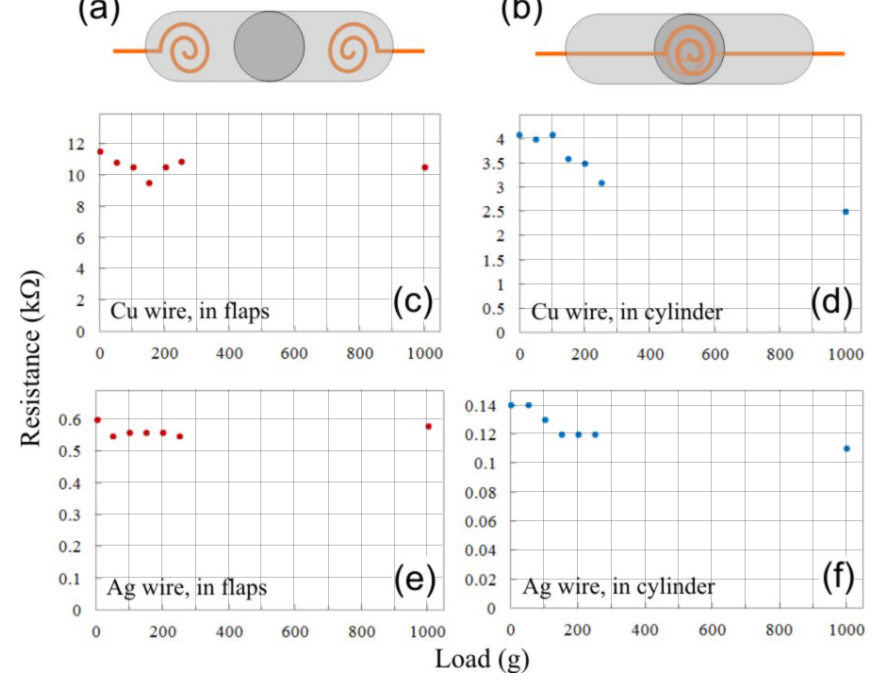

Fig. 3. Measured resistance vs load for the sample of the same shape as in Fig. 2a and two electrode wires copper and silver-plated copper and two electrode configurations in the flaps (outside the compression zone; a,c,e) and at the cylinder ends (within the compression zone; b,d,f).

In the next experiment, samples of the same shape as before were cast (AD-1 silicone $+10 \%$ w/w carbon black) with wire electrodes made of copper wire $(0.2 \mathrm{~mm}$ diameter) or silver-plated copper wire $(0.1 \mathrm{~mm}$ diameter $)$. The resistance vs load was measured with the electrodes in the flaps (i.e. unaffected by compressing the cylinder, Fig. 3 a,c,e) and with the electrodes in the cylinder (i.e. within the compression zone, Fig. 3 b,d,f). Again, with the wire electrode contacts not affected by compression, the tactel resistance changes by $25 \%$ (and not monotonically) for the copper wire and by $10 \%$ for the silver wire up to $1000 \mathrm{~g}$ load on the cylinder. At the same time, with the wires in the compression zone, the resistance decreases monotonically up to $250 \mathrm{~g}$ load. These results indicate that for the electrodes in the typical configuration used in piezoresistive pressure sensors i.e. embedded within the compression zone the electrical contacts between the tactel body (carbon-loaded silicone rubber) and the metal electrodes contribute to the piezoresistive response of the tactel. Notably, with the silverplated electrodes the overall resistance can be 20 times lower than for the bare copper wires.

\section{Tubular pressure sensor manufacturing}

The pressure sensor prototype has a form of a silicone tube (30 mm long, $5 \mathrm{~mm}$ inner and $7 \mathrm{~mm}$ outer diameter) with 32 cylindrical tactels arranged in 8 rows. The tactel array is read out with parallel and diagonal electrodes [26], [27]. Each tactel has two electrodes: one at the bottom (parallel) and one at the top (diagonal, Fig. 4a), made of silver-plated copper wire $(0.1 \mathrm{~mm}$ diameter, Scientific Wire Company). As a straight wire mesh significantly reduces the soft skin flexibility, the wire has an undulating shape with $0.6 \mathrm{~mm}$ width and $0.8 \mathrm{~mm}$ period (Fig. $4 \mathrm{~b}$ ).

Based on our previous findings, each of the 32 tactels was a $1.4 \mathrm{~mm}$ diameter cylinder made of carbon-loaded silicone (AD-1 with $10 \%$ w/w carbon black, Super P, Conductive, (a)

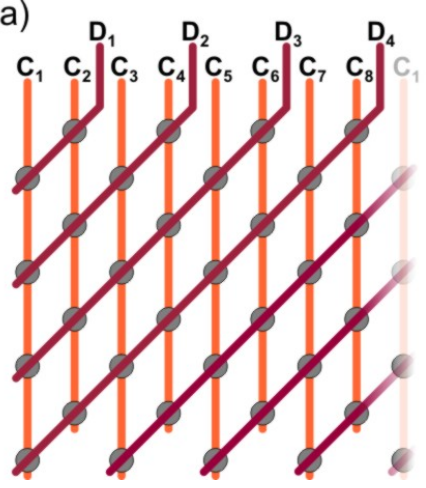

(b)

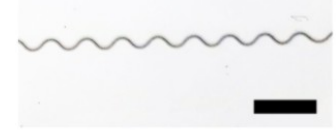

(c)

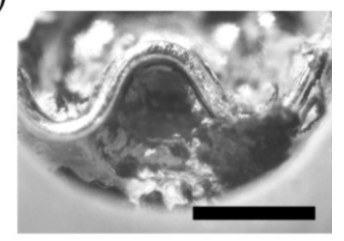

Fig. 4. Wire electrodes for the pressure-sensitive sleeve. (a) Electrode layout (shown on a plane) with 8 parallel (C1-8) and four diagonal (D1-4) electrodes. (b) Photograph of the undulating $0.1 \mathrm{~mm}$ diameter silver-plated copper wire used for the electrodes. Scale bar is $2 \mathrm{~mm}$ long. (c) Optical microscope photograph of the $1.4 \mathrm{~mm}$ diameter well, laser-milled in the silicone membrane with the wire electrode exposed at the bottom. Scale bar is $0.5 \mathrm{~mm}$ long.

(a)

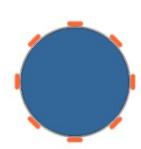

(e)

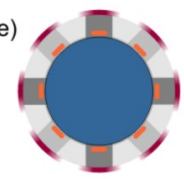

(b)
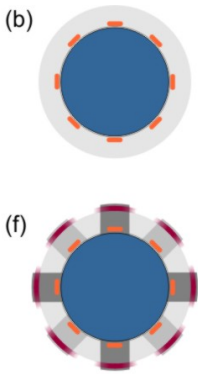

(c)
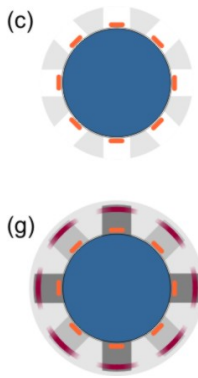

(d)
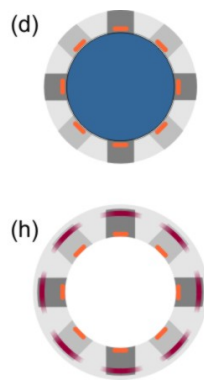

Fig. 5. Tubular pressure sensor fabrication steps. (a) A cylindrical mandrel (blue) is wrapped in aluminum foil and parallel electrodes (orange) are glued (temporarily) in place to the foil. (b) A $1 \mathrm{~mm}$ wall thickness silicone carrier tube (light gray) is cast. (c) $1.4 \mathrm{~mm}$ diameter tactel wells are lasermilled in the silicone tube. (d) The tactel wells are filled with conducting (carbon-loaded) silicone (dark gray). (e) The diagonal electrodes (purple) are wrapped around the silicone tube. (f) The top contacts are applied onto each tactel. (g) The outer silicone layer is applied. (h) The mandrel and aluminum foil are removed.

H30253, Alfa Aesar). The tactels were made by injecting the carbon-loaded silicone into cavities laser-milled in the silicone membrane.

To make the tubular pressure sensor, a $5 \mathrm{~mm}$ diameter PVC tube was wrapped in aluminum foil and 8 parallel electrodes were glued to the foil with a solvent-based glue (Bostik Soft Plastic Clear) (Fig. 5a and 6a). Centering rings were mounted on both ends of the tube before it was inserted into a two-part PMMA mould ( $7 \mathrm{~mm}$ bore diameter). A twopart silicone (Gumosil AD-1) was mixed 1:10 w/w with the platinum catalyst for 1-2 minutes and the mixture was degassed for 1-2 minutes in a vacuum chamber. The silicone mixture was injected into the mould with a syringe and left to cure overnight at room temperature (Fig. $5 b$ and 6b). After removing the tube from the mould, $321.4 \mathrm{~mm}$ diameter (nominal) wells were laser-milled (VLS2.30, Universal Laser Systems) to define the tactels (Fig. 5c and 6c). The silicone was removed down to the aluminum foil to expose the parallel electrode at the bottom of each well (Fig. 4c). Carbon-loaded silicone was injected into the wells (Fig. 5d 

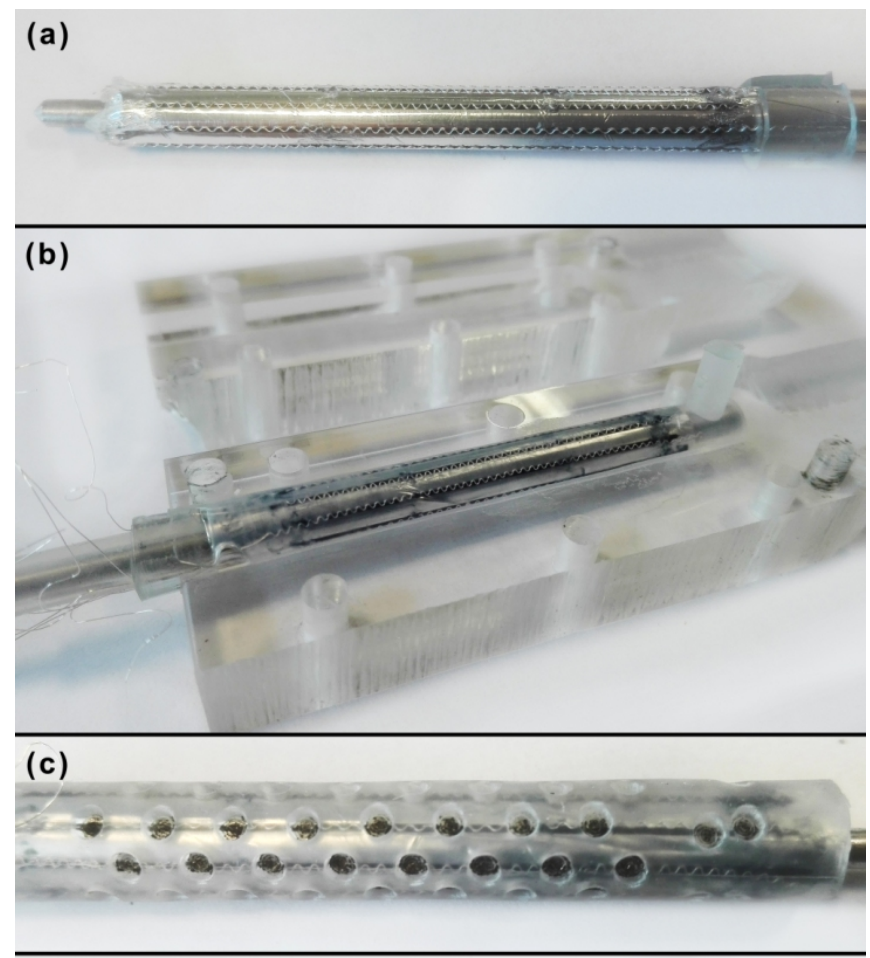

(d)

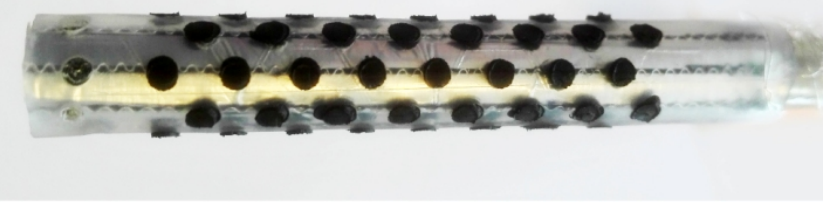

Fig. 6. Soft pressure sensor sleeve prototype fabrication. (a) The mandrel tube is wrapped in aluminium foil with parallel electrodes glued temporarily in place. One of the centring rings is visible on the right. (b) Silicone carrier membrane is cast in a PMMA mould. (c) The silicone tube with laser milled wells. (d) The wells after filling with carbon-loaded silicone.

and 6d) and once it cured overnight, the diagonal electrodes were wrapped around the tube (Fig. 5e). Small amount of carbon-loaded silicone was applied on top of each tactel to provide electrical contact with the top wire electrode (Fig. 5f) and after it cured overnight, the entire tube was painted over with the AD-1 silicone diluted with toluene $(1 \mathrm{~g}$ silicone + $0.1 \mathrm{~g}$ catalyst $+1 \mathrm{ml}$ toluene) that formed the outermost layer of approximately $0.2 \mathrm{~mm}$ thickness (Fig. $5 \mathrm{~g}$ ). Finally, the mandrel tube was removed and the sensor put in the acetone overnight to dissolve the glue and release the aluminum foil (Fig. 5h). The resistance of an individual tactel measured between the bottom and top wire electrodes with no force applied was $0.5 \pm 0.1 \mathrm{k} \Omega$.

Eight column electrodes were addressed in sequence via a multiplexer, and in every cycle the voltage on the four diagonal electrodes was measured. The multiplexer (MUX, DG509, Maxim) was driven with digital outputs of a USB DAQ card (USB-1608G, Measurement Computing) and the same card's four analog inputs sequentially read out the diagonal electrode potentials (Fig. 7). To avoid crosstalk between tactels, a virtual ground configuration with high-

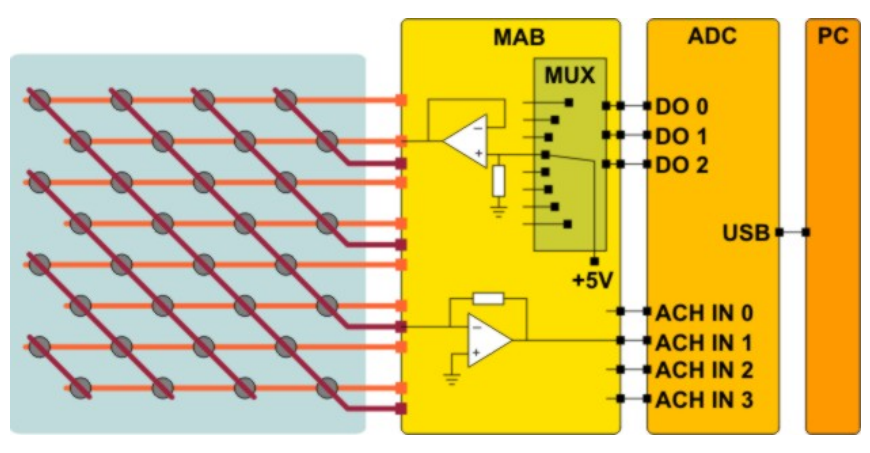

Fig. 7. Sensor array readout electronics. MAB - multiplexer and op-amp board, ADC analog and digital I/O card, DO 0-2 digital outputs, ACH IN 1-4 analog inputs, PC - personal computer. For clarity only one addressing line (out of eight) and one readout channel (out of four) are shown.

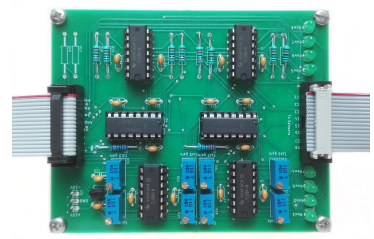

(a)

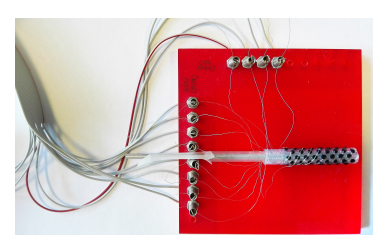

(b)
Fig. 8. (a) Custom made printed circuit board with the multiplexers and operational amplifiers for the sensor addressing and readout. (b) The pressure sensor prototype on the test board with electrical connections for 32 tactels 8 parallel and 4 diagonal electrodes.

gain operational amplifiers (TL084, Texas Instruments) [28] was used. The multiplexer and op-amps electronics board (Fig. 8a) had connections to the DAQ card and to the PMMA test board (Fig. 8b). Data acquisition, processing and visualisation were performed in LabView.

\section{Results AND Discussion}

Figure 9a presents the normalized, background-subtracted response of a single tactel, measured with a digital balance for a quasi-static load. The response is nonlinear, similar to this in Fig. 2c, measured with outside metal electrodes, indicating contribution from the changes in resistance of the silicone-metal contacts. The useful pressure range extends to around $400 \mathrm{kPa}$ (assuming uniform load onto the tactel area), above which the sensor saturates.

Measured single tactel transient response to an impulsive load is plotted in Fig. 9b where rise and fall times of around $50 \mathrm{~ms}$ are visible. Figure 9c shows the 48 sensor array background-subtracted readout with $620 \mathrm{kPa}$ pressure applied to one tactel.

We demonstrated the design, fabrication and preliminary characterization of a flexible, soft pressure sensing sleeve using medical grade materials. Bio-compatible silicone rubbers can be processed in millimeter-scale with precision casting and laser cutting/milling. Before a reliable sensor arrays can be made and used a number of phenomena need to be studied in more depth, including the processes involved in the piezoresistive response of the bulk materials, the role of electrode/silicone contacts and the parameters responsible for the sensor fabrication reliability and repeatability. For 

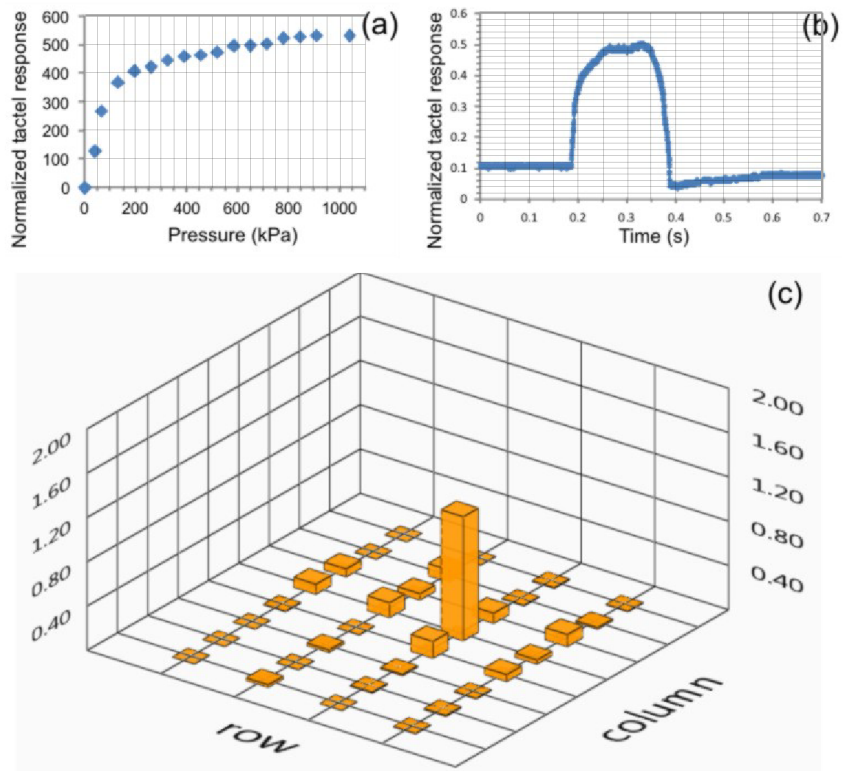

(c)

Fig. 9. The tubular pressure sensor response. (a) Measured response of a single tactel to a quasi-static pressure. (b) Measured transient response of a single tactel to an impulsive load. The horizontal scale spans $700 \mathrm{~ms}$, the rise and fall times are around $50 \mathrm{~ms}$. (c) Snapshot of the $4 \times 8$ sensor array with $620 \mathrm{kPa}$ pressure applied to one tactel.

a stretchable sensing skin for a tubular robot some of the remaining challenges are to optimise parameters such as tactel diameter, thickness and material composition as well as decouple the influence of the skin deformation from the external pressure readout. One way to address this later issue would be to retrieve the skin deformation from the known pose (shape) of the flexible robot and account for it in the pressure sensor data post-processing. Despite all of these we have shown a potential solution including fabrication of a flexible skin sensor for a tubular configuration that we believe has a role in future medical robots.

\section{REFERENCES}

[1] J. ONeill, J. Lu, R. Dockter, and T. Kowalewski, "Stretchable, flexible, scalable smart skin sensors for robotic position and force Estimation", Sensors, vol. 18, no. 4, pp. 953, 2018.

[2] J. M. Engel, et al., "Multi-layer embedment of conductive and nonconductive PDMS for all-elastomer MEMS", IEEE Int. Conf. MicroElectromechanical Systems, pp. 246-250, 2006.

[3] M. A. Qasaimeh, S. Sokhanvar, J. Dargahi, M. Kahrizi, "PVDFBased microfabricated tactile sensor for minimally invasive surgery", J. Microelectromechanical Systems, vol. 18, no. 1, pp. 195-207, 2009.

[4] P. Alpuim, et al., "Piezoresistive silicon thin film sensor array for biomedical applications", Thin Solid Films, vol. 519, no. 14, pp. 45744577, 2011.

[5] S. Stassi, V. Cauda, G. Canavese, and C. F. Pirri, "Flexible tactile sensing based on piezoresistive composites: A review", Sensors, vol. 14, no. 3, pp. 5296-5332, 2014.

[6] Y. Zang, F. Zhang, C. Di, and D. Zhu, "Advances of flexible pressure sensors toward artificial intelligence and health care applications", Materials Horizons, vol. 2, no. 2, pp. 140-156, 2015.

[7] H. Mei, et al., "A flexible pressure-sensitive array based on soft substrate", Sensors and Actuators A: Physical, vol. 222, pp. 80-86, 2015.

[8] T. D. Nguyen, H. S. Han, H.-Y. Shin, C. T. Nguyen, H. Phung, H. V. Hoang, and H. R. Choi, "Highly sensitive flexible proximity tactile array sensor by using carbon micro coils", Sensors and Actuators A: Physical, vol. 266, pp. 166-177, 2017.
[9] B. Nie, R. Li, J. D. Brandt, and T. Pan, "Microfluidic tactile sensors for three-dimensional contact force measurements", Lab on a Chip, vol. 14, no. 22, pp. 4344-4353, 2014.

[10] P. J. Codd, A. Veaceslav, A. H. Gosline, and P. E. Dupont, "Novel pressure sensing skin for detecting impending tissue damage during neuroendoscopy", Journal of Neurosurgery: Pediatrics, vol. 13, no. 1, pp. 114-121, 2014.

[11] R. D. P. Wong, J. D. Posner, and V. J. Santos, "Flexible microfluidic normal force sensor skin for tactile feedback, Sensors and Actuators A: Physical", vol. 179, pp. 62-69, 2012.

[12] D. M. Vogt, Y. Park, and R. J. Wood, "Design and characterization of a soft multi-axis force sensor using embedded microfluidic channels", IEEE Sensors Journal, vol. 13, no. 10, pp. 4056-4064, 2013.

[13] S. Gong, et al., "A wearable and highly sensitive pressure sensor with ultrathin gold nanowires", Nature Communications, vol. 5, pp. 3132, 2014.

[14] L. Wang, et al., "PDMS/MWCNT-based tactile sensor array with coplanar electrodes for crosstalk suppression", Microsystems \& $\mathrm{Na}$ noengineering, vol. 2, pp. 16065, 2016.

[15] A. D. Valentine et al., "Hybrid 3D printing of soft electronics", Adv Materials, vol. 29, no. 40, pp. 1703817, 2017

[16] M. Shimojo, A. Namiki, M. Ishikawa, R. Makino, and K. Mabuchi, "A tactile sensor sheet using pressure conductive rubber with electrical wires stitched method", IEEE Sensors Journal, vol. 4, no. 5, pp. 589596, 2004.

[17] Y. R. Wang, et al., "A flexible piezoelectric force sensor based on PVDF fabrics", Smart Mater. Struct., vol. 20, pp. 045009, 2011.

[18] M.-A. Lacasse, V. Duchaine, and C. Gosselin, "Characterization of the electrical resistance of carbon-black-filled silicone: Application to a flexible and stretchable robot skin", IEEE Int. Conf. Robotics and Automation, 2010.

[19] D. Z. M. Ramirez, et al., "Characterization of bespoke force sensors for tailored applications", IEEE Sensors J., vol. 17, no. 6, pp. 1727 $1735,2017$.

[20] A. Chortos, J. Liu and Z. Bao, "Pursuing prosthetic electronic skin", Nature Materials, vol. 15, pp. 937950, 2016.

[21] D. Beruto, M. Capurro, and G. Marro, "Piezoresistance behavior of silicone -graphite composites in the proximity of the electric percolation threshold", Sensors and Actuators A: Physical, vol. 117 pp. 301-308, 2005.

[22] L. Wang, T. Ding, and P. Wang, "Effects of instantaneous compression pressure on electrical resistance of carbon black filled silicone rubber composite during compressive stress relaxation", Composites Science and Technology, vol. 68, pp. 3448-3450, 2008.

[23] L. Wang, T. Ding, and P. Wang, "Research on stress and electrical resistance of skin-sensing silicone rubber/carbon black nanocomposite during decompressive stress relaxation", Smart Materials and Structures, vol. 18, no. 6, pp. 065002, 2009.

[24] J. Kost, M. Narkis, and A. Foux, "Resistivity behavior of carbon black- filled silicone rubber in cyclic loading experiments", J. Applied Polymer Science, vol. 29, no. 12, pp. 3937-3946, 1984.

[25] W. Min, H. Ying,G. Yunjian, F. Xiulan, and H. Panfeng, "A study of resistance relaxation phenomenon based on carbon black/silicone rubber system flexible tactile sensor", Int. Conf. Information Acquisition, pp. 366-371, 2007.

[26] R. S. Saxena, R. K. Bhan, and A. Aggrawal, "A new discrete circuit for readout of resistive sensor arrays", Sensors and Actuators A: Physical, vol. 149 , no. 1, pp. 93-99, 2009.

[27] T. D'Alessio, "Measurement errors in the scanning of piezoresistive sensors arrays", Sensors and Actuators A: Physical, vol. 72, no. 1, pp. 71-76, 1999.

[28] R. S. Saxena, R. K. Bhan, N. K. Saini, and R. Muralidharan, "Virtual ground Technique for crosstalk suppression in networked resistive sensors”, IEEE Sensors J., vol. 11, no. 2, pp. 422-433, 2011. 